\title{
A Beta Regression Model for Himalayan Medicinal Plant Disease Prediction
}

\author{
RadhikaSharma ${ }^{1 *}$, V. D. Shivling ${ }^{2}$, Dinesh Kumar ${ }^{1}$, Atul Kumar Sharma ${ }^{3}$ \\ 'Punjab Technical University, Punjab, India; radhika489@ymail.com \\ ${ }^{2}$ CSIR-CSIO Chandigarh, India \\ ${ }^{3}$ NITKurukshetra, Haryana, India
}

\begin{abstract}
Picrorhiza kurrooa plants were grown in greenhouse and was tested for powdery mildew disease. The result of temperature and wetness duration on P.kurrooa was studied under controlled-environment to develop a disease prediction model for controlling infection.Plants were kept at a temperature ranging from $31^{\circ} \mathrm{C}$ to $37^{\circ} \mathrm{C}$ with Relative Humidity (RH) more than $90 \%$ was maintained in greenhouse and it is measured in the terms of wetness duration (in hours) from 5 to $40 \mathrm{hr}$.To determine the relationship between infection index, temperature and wetness duration data for $P$. kurrooa were analyzed by nonlinear regression model. Beta model was used to provide the best fit to the data for modeling. Infection index on plant increased with increasing wetness duration at optimum temperature. Minimum and maximum temperatures for infection were around 31 and $37^{\circ} \mathrm{C}$, respectively. At $35.5^{\circ} \mathrm{C}$ maximum infection was recorded, and a minimum duration of wetness that is required for germination of the fungus was $5 \mathrm{hr}$. Highest infection index 0.95 was noticed at $35.5^{\circ} \mathrm{C}$. Temperature and duration of wetness were recorded for each event and used in the model equation to calculate disease infection index. Regression coefficient $\left(\mathrm{R}^{2}\right)$ between observed infection index and predicted infection index was 0.8103 and coefficient of determination (R) was 0.9 . It indicates that the model could reliably predict the disease infection index over a wide range of temperatures and wetness durations.
\end{abstract}

Keywords: Beta Regression Model, Picrorhiza kurrooa, Powdery Mildew Prediction System

\section{Introduction}

Picrorhiza kurrooa Royle ex Benth generally known as P. kurrooa is a perennial herb of medicinal value usually found at high altitude ranging from 3000-5000m amsl. P. kurrooa is dominant in Western Himalayan regions of Northern part of India while Picrorhiza scrophulariflora Pennel second species of Picrorhiza (Scrophulariaceae) is generally found in Eastern Himalayan regions of Sikkim, $\mathrm{Nepa}^{2,3}$. Aerial as well as underground parts (roots and rhizomes) contain picrosides, the medicinally important constituents of P. kurrooa ${ }^{10,11}$. P.kurrooa has many therapeutic uses. Picroside-I, Picroside-II and Kutkoside are important constituents of the species which have many medicinal uses. P. kurrooa is traditionally used for the intervention of liver disorders, fever, asthma and jaun- dice. Over exploitation of the species by the traders coupled with lack of cultivation has led the species to be in the list of endangered plants ${ }^{4,15}$. In order to conserve the species,various $e x$-situ and in-situ plantation system is being encouraged.Various approcahes are discussed ${ }^{8}$ to conserve this endangered species.

When P. kurrooa is brought to a lower altitude for cultivation attack of powdery mildew and white fly is inevitable even at early stages of plant growth ${ }^{12,13}$.

Fungicide application for controlling powdery mildew spots consists of 2-3 sprays after initiation. The incidence and index of powdery mildew are higher during high humidity and moderate temperature ${ }^{14}$. The infection index of disease is imputed to the variability in environmental conditions basically temperature and wetness duration. Sinha ${ }^{14}$ reported that fungus germinate at

${ }^{*}$ Author for correspondence 
temperature between $6^{\circ}$ to $33^{\circ} \mathrm{C}$ and become idle above $35^{\circ} \mathrm{C}$; at least $5 \mathrm{hr}$ of wetness duration at optimum temperature is required for infection to occur. Similarly, the influence of temperature and wetness duration on infection of Apple and strawberry flowers is discussed ${ }^{1,5}$ respectively. By modeling the influence of temperature and wetness duration forecasting system had already been developed for rice and onion ${ }^{9,16}$.

Therefore, there is a need to develop a disease forecasting system so as to inform the growers/farmers to adopt appropriate control measures well in advance to enable crop protection before infection. With this background, the present work is focused on (i) to find out the temperature and wetness duration required for powdery mildew to occur on P. kurrooa, and (ii) develop a prediction model for powdery mildew infection.

\section{Materials and Methods}

\subsection{Controlled Environmental Studies}

Symptomless cuttings of rhizomes (roots of the plant) of P. kurrooa were collected from the Rohtang pass (4000 $\mathrm{m}$ height), India. These cuttings were planted in the greenhouse and allowed to grow under controlled environmental conditions.

Continuous monitoring of growth of the plant was done. Data was generated under controlled conditions through vaccination of collected cuttings so as to determine the effects of temperature and leaf wetness duration (here leaf wetness duration means the count of duration (in hours) when humidity is greater than $90 \%$ ) on the plant infection. Using this data Beta regression model was developed to predict the risk of powdery mildew in the plant. For continuous monitoring and recording of temperature as well as duration of wetness, temperature sensor Probe Young (41382VC) and leaf wetness sensor model Decagon (LWS 40020) was used. Growth of fungus was evaluated at different temperature and wetness durations.

\subsection{Germination of Conidia}

Conidia germination was tested at temperature ranging from $31^{\circ} \mathrm{C}$ to $37^{\circ} \mathrm{C}$ by keeping $\mathrm{RH}$ constant at $90 \%$. Humidity of the greenhouse was maintained using saturated salt solution by following the method of Winston and Bates ${ }^{17}$. From the infected leaf showing growth of powdery mildew, a dust of conidia was prepared by scraping the infected leaf and kept on the dry glass slide which was kept in humid chamber and opened to the mentioned temperature range. Similarly, conidia germination was checked by varying the RH between 0 to $90 \%$ for different time duration of 5, 10, 15, 20, 24, 26, 30, 34 and $40 \mathrm{hr}$ by keeping the temperature of the chamber constant at $35.5^{\circ} \mathrm{C}$. As maximum germination was observed at $35.5^{\circ} \mathrm{C}$ so it was considered as optimum (ideal) temperature for germination.

\subsection{Effect of Temperature on Vaccinated Plant}

Fresh cuttings of rhizomes were inoculated with conidia dust using hairbrush and kept in a chamber maintained with $\mathrm{RH}$ between $80-90 \%$ and temperature was maintained for each point of range from $31^{\circ} \mathrm{C}$ to $37^{\circ} \mathrm{C}$. The plant was kept in a chamber for $40 \mathrm{hr}$ and then exposed to atmospheric conditions having less than $90 \% \mathrm{RH}$ for several hours. Infection was observed in all the replications and infection index was calculated using the method used $^{14}$.

\subsection{Effect of Wetness duration on Vaccinated Plant}

Fresh cuttings of rhizomes were inoculated with conidia dust using hairbrush and kept in a growth chamber which was maintained with $\mathrm{RH}$ between $80-90 \%$, exposed for the time period of $5,10,15,20,24,26,30,34$ and $40 \mathrm{hr}$ at temperature $35.5^{\circ} \mathrm{C}$. Plants were exposed to atmospheric conditions for many hours. Infection index was then calculated from these replications and infection was observed in all the replicates.

\subsection{Analysis of Data and Model Development}

In order to (i) determine if temperature, wetness duration and their combination significantly affect the infection proportion of the plant and (ii) find a relationship between disease infection index and infection proportion of a leaf of the plant and environmental parameters that is temperature and wetness duration a regression analysis was used. For each wetness duration, temperature and their combination, different number of cutting of plants were considered. The infection index of sub-samples were determined for each temperature and wetness duration. Separate (for each temperature and 
wetness duration)as well as combined analysis for three replications were conducted.

Generalized form of Beta model ${ }^{6,7}$ is used for finding functional form of relationship between infection index and environmental parameters. The model can be written as

$$
Y=a t^{b}(1-t)^{c} W^{d}
$$

Here $\mathrm{Y}$ is proportion of plant infected or infection index, $\mathrm{W}$ is wetness duration (in hours), $\mathrm{a}, \mathrm{b}, \mathrm{c}$ and $\mathrm{d}$ are the unknown parameters and $t$ is temperature which can be written as:

$$
t=\frac{\left(T-T_{\min }\right)}{\left(T_{\max }-T_{\min }\right)}
$$

where, $T_{\min }$ and $T_{\max }$ are minimum and maximum temperature required for infection to occur respectively. And $\mathrm{T}$ is the current temperature at which $\mathrm{Y}$ has to be calculated. According to Erincik et al. ${ }^{6}$ in Eq. 1 b indicates that $\mathrm{Y}$ will increase steeply with an increase in $\mathrm{T}$ up to optimum temperature. The parameter $\mathrm{c}$ indicates that $\mathrm{Y}$ will decrease as T increases. Eq. 1 can be written as:

$$
\log (Y)=\log (a)+b \log (t)+c \log (1-t)+d \log (W)
$$

The model was evaluated using the following parameters:- (i) correlation coefficient (R), (ii) coefficient of determination $\left(\mathrm{R}^{2}\right)$, and (iii) Mean Square Error (MSE)

$$
\text { Mean Square Error }(\mathrm{MSE})=\frac{1}{n} \sum_{j-1}^{n}\left(\widehat{Y}_{j}-Y_{j}\right)^{2}
$$

where, $\widehat{Y}_{\rho}$ the predicted value at $j$ input; $Y_{j}$ is the target or actual value at $j$ input; and $n$ is the total number of inputs.

\section{Results}

With an increase in wetness (RH) duration at all temperatures, an increase in infection index was noticed. As the temperature increases from $31^{\circ} \mathrm{C}$ to $36^{\circ} \mathrm{C}$, the infection index increased steeply (Figure 1).

The infection index declined with further increase in temperature. At an ideal or optimum temperature of $35.5^{\circ} \mathrm{Cinfection}$ index was 0.95 (Figure 2).

0.95 was the maximum value of infection index. The index value declined to 0.85 and 0.22 at $36^{\circ} \mathrm{Cand} 37^{\circ} \mathrm{C}$, respectively at wetness duration of $40 \mathrm{hr}$. This reflected that further increase in temperature will result in substantial reduction in infection.
As we can see in the Figure 3 that the index value increased from 0.001 to 0.95 as wetness duration increases from 5 to $40 \mathrm{hr}$,respectively for $35.5^{\circ} \mathrm{C}$ which is the optimum temperature. Using this experimental data the values of unknown parameters of the generalized Beta model were calculated. As we can see in equation 1 the model includes two environmental parameters that are temperature and wetness duration and their product. The value of unknown parameters of the Beta model depends on these two environmental parameters and their value will be greater than 0 , therefore, disease severity is affected by the

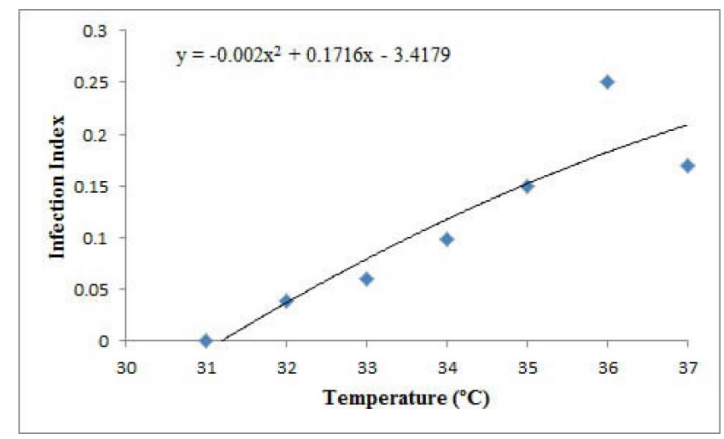

Figure 1. Response of powdery mildew infection on temperature at wetness duration of $5 \mathrm{hr}$.

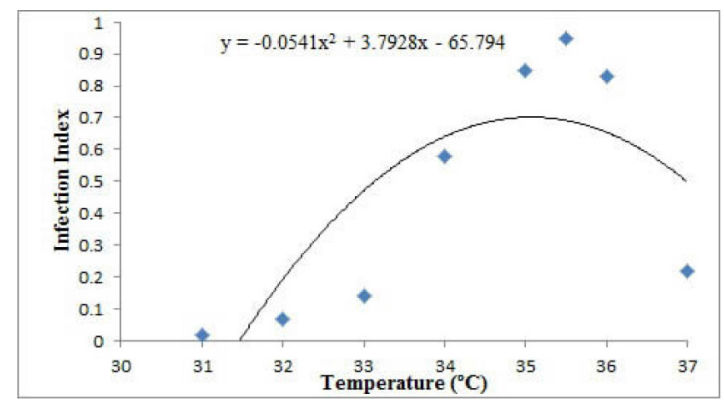

Figure 2. Response of powdery mildew infection on temperature at wetness duration of $40 \mathrm{hr}$.

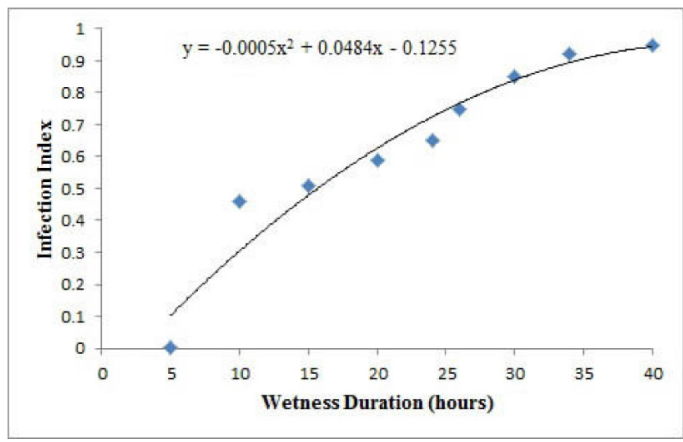

Figure 3. Response of Powdery mildew infection on hour of relative humidity (>90\%) at optimum temperature $35.5^{\circ} \mathrm{C}$. 
interaction between these two environmental parameters. Replication of the experiment was done so as to check for the variation in the value of parameters. Although there was a slight difference in the value of parameters but model fits to the data in all the replicates.

For generalized Beta model $T_{\min }$ and $T_{\max }$ should be known so as to determine the parameters of the Beta model. Using the data collected through experiments and Figure $1, T_{\min }$ was around $31^{\circ} \mathrm{C}$ and $T_{\text {max }}$ was slightly above $37^{\circ} \mathrm{C}$. For model development we used $T_{\min }=31 \pm$ $0.1^{\circ} \mathrm{C}$ and $T_{\max }=37 \pm 0.1^{\circ} \mathrm{C}$. The collected data was used to find the value of various unknown parameters whose value will be constant to form a standard equation for P.kurrooa.

As shown in Table 1 the value of all the parameters of the generalized Beta model is given for the various replications along with the coefficient of determination and the coefficient of correlation. The table includes the value of parameters for the three replications. The value of $\mathrm{R}^{2}$ for first replication was 0.81 which was more than as compare to the second and third replication that is 0.78 and 0.76 (Table 1). It is considered that for model fitness the value of $\mathrm{R}^{2}$ should be close to 1 . For this experiment the maximum value of the $\mathrm{R}^{2}$ was 0.81 . So we can say that the parameter value for replication 1 gives a more accurate value than the replication 2 and 3 .

Figure 4 indicates the relationship between the observed infection index values and the predicted infection index values. This graph indicates the value of $R^{2}$ that is 0.81 . Coefficient of determination represents how well data points fit the line. For the initial value of

Table 1. Evaluated parameters of the generalized Beta model for examining the effect of temperature and wetness duration on the P. kurroo $^{\mathrm{b}}$ infected by Powdery mildew

\begin{tabular}{|c|c|c|c|c|c|c|}
\hline & \multicolumn{4}{|c|}{$\begin{array}{c}\text { Value of unknown } \\
\text { parameters }^{\mathrm{a}}\end{array}$} & \multirow[b]{2}{*}{$\mathrm{R}^{2}$} & \multirow[b]{2}{*}{$\mathrm{R}$} \\
\hline & $\mathbf{a}$ & b & c & d & & \\
\hline Replication 1 & -7.43 & 1.18 & 0.41 & 2.23 & 0.81 & 0.9 \\
\hline Replication 2 & -5.23 & 1.56 & 1.15 & 3.01 & 0.78 & 0.88 \\
\hline Replication 3 & 1.68 & 1.03 & 0.91 & 2.87 & 0.76 & 0.87 \\
\hline \multicolumn{7}{|c|}{$\begin{array}{l}\text { aValue of parameters is estimated using the Beta model equation : } \mathrm{a} \text { is evaluated } \\
\text { from log-transformed } \mathrm{a}, \mathrm{b} \text { corresponds to the effect of temperature and } \mathrm{d} \\
\text { corresponds to the effect of wetness duration. For calculating the value of } \\
\text { these parameters software approach was used and programming was done in } \\
\text { MATLAB. } \\
{ }^{\circ} \mathrm{R} 2 \text { (Coefficient of determination) and } \mathrm{R} \text { (coefficient of correlation) are } \\
\text { evaluated for each replication. }\end{array}$} \\
\hline
\end{tabular}

infection index, fitness of the data points is better than for the higher value of infection index.

Figure 5 shows the residual plot between observed and predicted values. It basically represents the difference between the observed and predicted value at a particular temperature and wetness duration.

\section{Discussion}

Powdery mildew usually occurs in the month of MayJune. It causes great loss to the P.kurrooa. The conidia germination starts under favorable environmental conditions and is related to the duration of wetness. As the wetness increased, infection index also increased. For every pest and disease development, there is a favorable temperature range; before and after that range the germination is negatively impacted ${ }^{14}$. For powdery mildew of P.kurrooa, the infection starts decreasing after $37^{\circ} \mathrm{C}$.

The model can be used for early forecasting of disease and a software tool can be used for fast and automatic calculation and simulation of the infection index. Forecasting system for powdery mildew of P.kurrooa has not yet developed. This model gives the best fitness between the

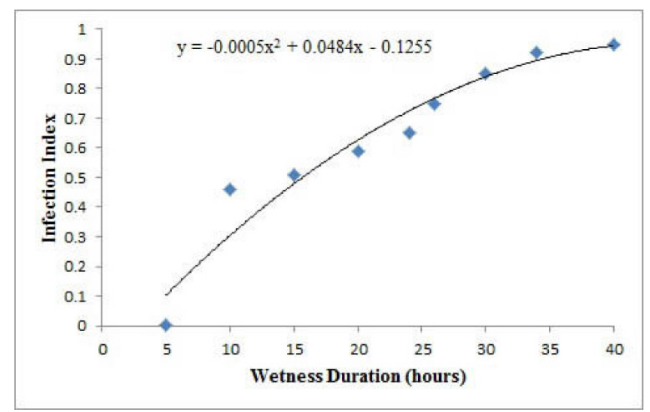

Figure 4. Model Validation- closeness between observed and predicted infection index value.

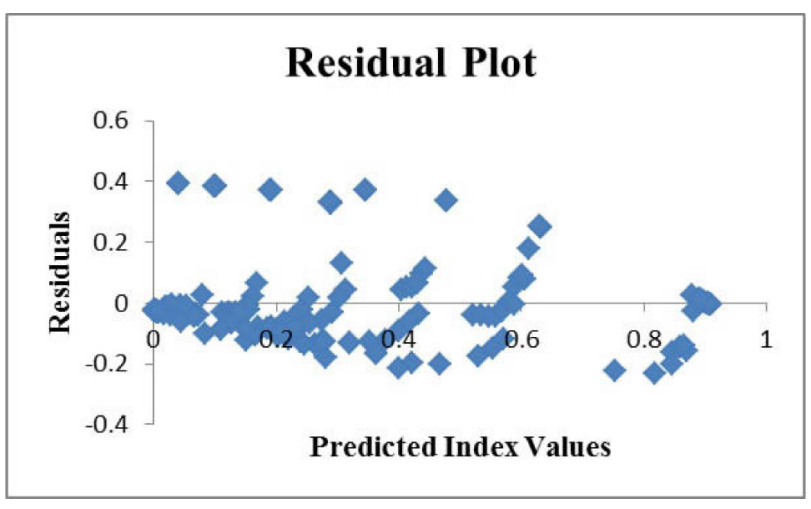

Figure 5. Residual plot for the predicted infection index. 
observed value and predicted value. The high value of the correlation coefficient and the coefficient of determination denote that this model approaches the real value of observation and gives the best approximation. Data point distribution in the residual graph shows that this model is suitable for the nonlinear observation points. For the forecasting of the disease, it gives the suitable solution and a system or machine can be easily trained through particular software using this model.

\section{References}

1. Arauz LF, Sutton TB. Temperature and wetness duration requirements for apple infection by Botryosphaeria obtuse. Phytopathology. 1989;79(4):440-444.

2. Bantawa P, Ghosh SK, Bhandari P, Singh B, Ghosh PD, Ahuja PS, Mondal TK. Micropropagation of an elite line of Picrorhiza scrophulariiflora, Pennell, an endangered high valued medicinal plant of the Indo-China Himalayan regions. Med Arom Plant Biotech. 2010; 4:1-7.

3. Bantawa P, Saha-Roy O, Ghosh SK, Mondal TK. In vitro regeneration of an endangered medicinal plant Picrorhiza scrophulariiflora. Biologia Plantarum. 2011; 55(1):169-72.

4. Behera MC, Raina R. Gentiana kurroo Royle-A critically endangered bitter herb. International Journal of Medicinal and Aromatic Plants. 2012; 2(1):22-9.

5. Bulger MA, Ellis MA, Madden LV. Influence of temperature and wetness duration on infection of strawberry flowers by Botrytis cinerea and disease incidence of fruit originating from infected flowers. Phytopathology. 1987; 77.

6. Erincik O, Madden LV, Ferree DC, Ellis MA. Temperature and wetness-duration requirements for grape leaf and cane infection by Phomopsis viticola. Plant Disease. 2003; 87(7):832-40.

7. Grove GG, Madden LV, Ellis MA, Schmitthenner AF. Influence of temperature and wetness duration on infection of immature strawberry fruit by Phytophthora cactorum. Phytopathology. 1985; 75(2):165-69.

8. Jain M, Johnson TS, Krishnan P. Biotechnological approaches to conserve the wealth of nature: endangered and rare medicinal plant species, a review. Journal of Natural Remedies. 2012; 12(2):93-102.

9. Kapoor A, Prasad R, Sood G. Forecasting of rice blast in Kangra district of Himachal Pradesh. Indian. Phytopathology. 2012; 57.

10. Kumar R, Bhandari P, Singh B, Ahuja PS. Evaluation of Picrorhiza kurroa accessions for growth and quality in north western Himalayas.J Med Plants Res. 2012; 6:2660-65.

11. Mehra TS, Chand R, Sharma Y. Reproductive biology of Picrorhiza kurroa-a critically endangered high value temperate medicinal plant.Open Access Journal of Medicinal and Aromatic Plants. 2011; 1(2):40-3.

12. Ramdas GK, Pokhriyal P. Seeds germination and seedlings analysis of Picrorhiza kurrooa royle ex benth. in Genwala and Bagori (Harsil) of district Uttarkashi (Uttarakhand); 2012.

13. Sharma R. Agro-techniques of medicinal plants. Daya Publishing House; 2004.

14. Sinha P. Epidemiology and development of forecasting model for powdery mildew (Oidium erysiphoides) in jujube (Ziziphus mauritiana\} for Delhi. Indian J Agr Sci. 2005; 75(5):272-76.

15. Jan A, Thomas G, Shawl AS, Jabeen N, Kozgar MI. Improved micropropagation protocol of an endangered medicinal plant-Picrorhiza Kurroa Royle ex Benth. promptly through auxin treatments. Chiang Mai J Sci. 2010; 37(2):304-13.

16. Vincelli PC, Lorbeer JW. BLIGHT-ALERT: A weatherbased predictive system for timing fungicide applications on onion before infection periods of Botrytis squamosa. Phytopathology, 1989; 79(4), 493-498.

17. Winston PW, Bates DH. Saturated solutions for the control of humidity in biological research. Ecology. 1960; 41(1):232-37. 